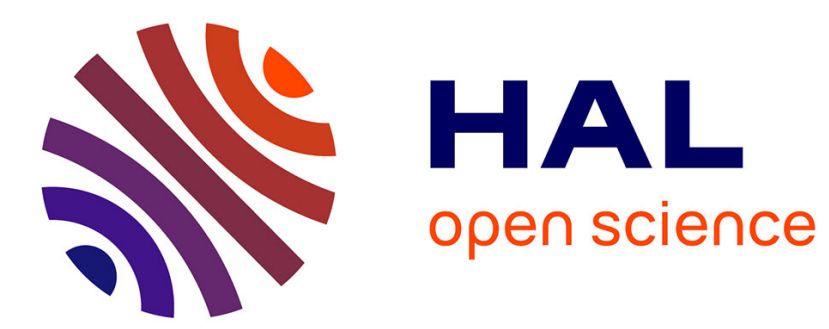

\title{
SOLITONS ACOUSTIQUES GUIDÉS DANS UNE PLAQUE CRISTALLINE À SYMÉTRIE CUBIQUE
}

B. Collet

\section{To cite this version:}

B. Collet. SOLITONS ACOUSTIQUES GUIDÉS DANS UNE PLAQUE CRISTALLINE À SYMÉTRIE CUBIQUE. Journal de Physique IV Proceedings, 1992, 02 (C1), pp.C1-733-C1-736. 10.1051/jp4:19921159 . jpa-00251118

\section{HAL Id: jpa-00251118 https://hal.science/jpa-00251118}

Submitted on 1 Jan 1992

HAL is a multi-disciplinary open access archive for the deposit and dissemination of scientific research documents, whether they are published or not. The documents may come from teaching and research institutions in France or abroad, or from public or private research centers.
L'archive ouverte pluridisciplinaire HAL, est destinée au dépôt et à la diffusion de documents scientifiques de niveau recherche, publiés ou non, émanant des établissements d'enseignement et de recherche français ou étrangers, des laboratoires publics ou privés. 


\title{
SOLITONS ACOUSTIQUES GUIDES DANS UNE PLAQUE CRISTALLINE À SYMETRIE CUBIQUE
}

\author{
B. COLLET \\ Laboratoire de Modélisation en Mécanique, associé au CNRS URA 229, Université Pierre et Marie \\ Curie, Tour 66, Boîte 162, 4 place Jussieu, F-75252 Paris cedex 05, France
}

\begin{abstract}
Dispersion and nonlinearity are two necessary ingredients for the existence in physical systems of solitons. In the present work the influences of dispersions and of nonlinearity material on shear horizontal $(S H)$ waves in simple-cubic crystal plates with free faces are examined on basis of nonlinear equations extracted from 3-dimensional lattice model. The analysis is restricted to excitations which consist of a slowly varying envelope modulating a harmonic carrier which is not limited to long wave length. In the case of excitations of small amplitude it is shown that the amplitude of the envelope is governed by a cubic nonlinear Schrödinger equation.
\end{abstract}

\section{1. - INTRODUCTION}

Les excitations non linéaires localisées en espace-temps qui se propagent dans les systèmes physiques à vitesse constante en conservant presque indéfiniment leur forme initiale sont nommés ondes solitaires ou solitons nécessitent une rigoureuse compensation entre deux caractéristiques antagonistes non nonlinéarité et dispersion. Les solitons se manifestent principalement sous forme : de fronts d'onde ; d'impulsions du type bosse ; de modes de respiration et d'enveloppes. Les solitons enveloppes ont été observés expérimentalement dans plusieurs branches de la physique comme : l'hydrodynamique, la physique des plasmas, d'optique non linéaire, les transmissions par ligne électrique et l'acoustoélasticité [1-4]. Le transport de l'information s'effectue généralement par modulation (amplitude, fréquence, phase) d'une onde porteuse, la classe de solitons la mieux adaptée au développement d'applications dans ce domaine est naturellement celle des solitons enveloppes.

L'objet de ce travail est d'étudier les effets de la non linéarité matérielle et des dispersions (géométrique et intrinsèque) et de leur compensation parfaite sur la propagation d'ondes de cisaillement à polarisation horizontale $(S H)$ dans une plaque élastique à symétrie cubique simple (fig. 1). Dans ce but on construit un modèle de réseau cristallin tridimensionnel où les particules interagissent avec les premières et deuxièmes voisines (fig. 2) via des potentiels de couplage élastiques quartiques. Sur la base du modèle retenu on analyse la propagation d'excitations faiblement non linéaires ayant la forme d'un paquet ou d'un train d'ondes de cisaillement constitué par une enveloppe modulant une onde porteuse quasi-harmonique dont le vecteur d'onde n'est pas limité au strict voisinage de l'origine de la première zone de Brillouin. En dissociant l'étude de l'onde porteuse de celle de son enveloppe, on montre que l'amplitude de cette dernière est gouvernée par une équation de Schrödinger non linéaire de type cubique, dans laquelle le terme non linéaire dépend du nombre d'onde de la porteuse du coefficient matériel non linéaire, du mode considéré 
et de l'épaisseur de la plaque. Les solutions du problème posé, dans le cas de solitons obscurs sont données sous forme analytique et illustrées numériquement pour le mode $(S H)$ symétrique le plus bas (fondamental).

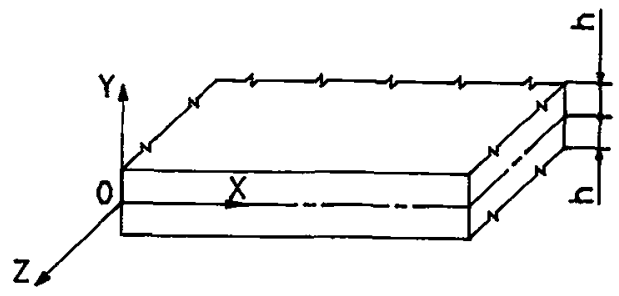

Fig.1 : Système de coordonnées pour une plaque cristalline d'épaisseur $2 h$

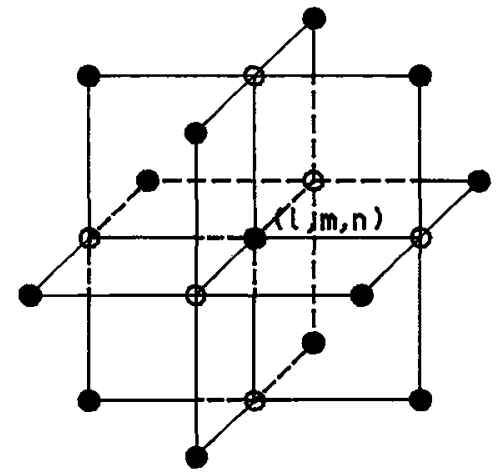

Fig.2 : Sites occupés par les premiers et deuxièmes voisins de l'atome $(\ell, m, n)$

\section{2. - MODELE ET EQUATIONS DISCRETES}

On considère un réseau cristallin tridimensionnel à symétrie cubique simple [5] formé d'atomes identiques de masse $M$. Chaque atome de cet édifice est soumis aux actions centrales de ses six premiers et douze seconds voisins représentées par des potentiels d'interactions non linéaires quartiques. Dans le cas traité on s'intéresse à la propagation d'ondes de cisaillement pur à polarisation horizontale $(S H)$ de direction de propagation [100] dans une plaque de cristal à plan médian (010) (fig. 1). On suppose donc que la position actuelle d'une particule $(\ell, m, n)$ du réseau est donnée par $X=\ell a, Y=m a, Z=n a+W(\ell, m ; t)$ où $\ell, m, n$ sont des entiers ; on désigne par $a$ la taille de la maille cristalline et $W$ le déplacement transversal dans la direction $Z$. Après une série de calculs les équations discrètes du mouvement et les conditions aux limites pour une plaque cristalline d'épaisseur $2 h=2 H a$ (avec $H$ entier positif), libre d'efforts sur ses faces, s'écrivent :

$$
\begin{aligned}
M \ddot{W}(\ell, m)= & A[(W(\ell+1, m)-2 W(\ell, m)+W(\ell-1, m))+(W(\ell, m+1)-2 W(\ell, m) \\
& +W(\ell, m-1))]+B\left[(W(\ell+1, m)-W(\ell, m))^{3}-(W(\ell, m)-W(\ell-1, m))^{3}\right. \\
& \left.+(W(\ell, m+1)-W(\ell, m))^{3}-(W(\ell, m)-W(\ell, m-1))^{3}\right] \\
& \left(W(\ell, \pm(H+1)-W(\ell, \pm H))\left(A+B(W(\ell, \pm(H+1))-W(\ell, \pm H))^{2}\right)=0\right.
\end{aligned}
$$

$A$ et $B$ sont respectivement des coefficients matériels linéaire et non linéaire qui s'expriment en fonction de coefficients des potentiels d'interactions impliqués et de la maille $a$.

Les relations de dispersion linéaires pour les modes $(S H)$ symétriques se déduisent de (1) en posant $B=0$ et en substituant le déplacement harmonique $W(\ell, m)=W_{0} \cos q m a \exp i(k \ell a-\omega t)$ où $k$ est un nombre d'onde et $\omega$ une fréquence circulaire ; on obtient

$$
\begin{gathered}
\bar{\omega}=\omega / \omega_{1 S V}=(2(2 H+1) / \pi)\left[\sin ^{2} \frac{1}{2} q a+\sin ^{2} \frac{1}{2} k a\right]^{1 / 2}, q=p \pi /(2 H+1), \\
p=0,2,4,6, \ldots \leq 2 H, 0 \leq k a \leq \pi, \omega_{1 S V}=\pi V_{T} /(2 H+1) a, V_{T}=\left(A a^{2} / M\right)^{1 / 2}
\end{gathered}
$$


où $\bar{\omega}$ sont des fréquences normalisées, $\omega_{1} S V$ une fréquence de référence et $V_{T}$ la vitesse acoustique des ondes de volume transversales. On note que les courbes de dispersion (fig. 3) présentent à l'origine et en bout de la première zone de Brillouin des fréquences de coupure. Par ailleurs et contrairement au cas d'une description continue le nombre de modes symétriques est ici fini.

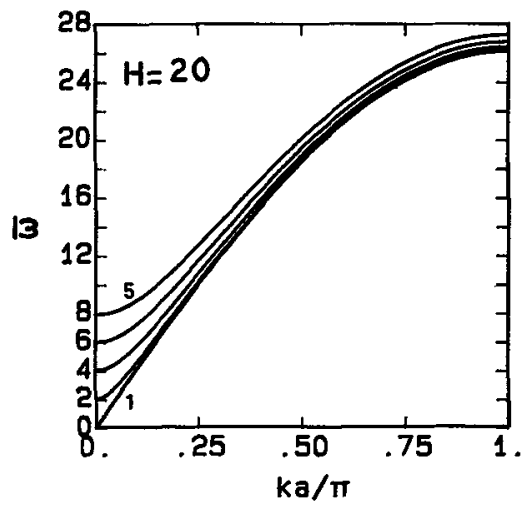

Fig.3 : Spectre linéaire des fréquences pour les 5 premiers modes $(S H)$ symétriques

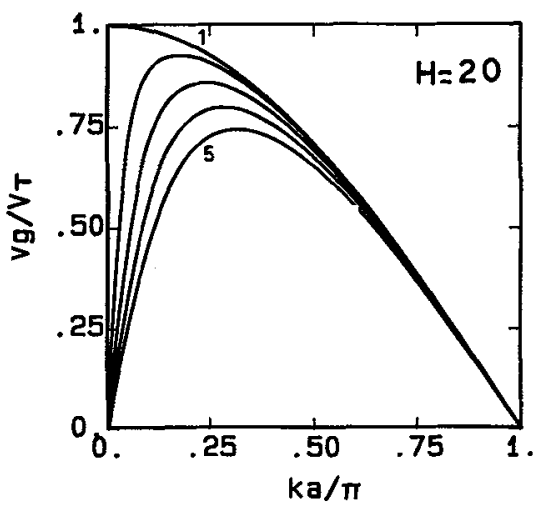

Fig.4 : Vitesse de groupe normalisée pour les 5 premiers modes $(S H)$ symétriques

\section{3. - EQUATION DE SCHRODINGER NON LINEAIRE ET SOLITONS OBSCURS}

Dans cette partie, on présente quelques résultats préliminaires concernant la propagation de solitons $(S H)$ dans une plaque de cristal à symétrie cubique simple [5]. Plus précisément on analyse l'évolution d'excitations non linéaires du type onde modulée. Une telle étude peut s'effectuer dans le cadre des milieux continus [6], mais dans ce cas les résultats ne sont valables que pour des excitations dont la porteuse est à variation lente (grande longueur d'onde) et l'enveloppe de grande extension spatiale. En conséquence il est donc préférable de résoudre le problème posé directement à partir du système discret (1) en utilisant l'approximation semi-discrète [7]. Cette méthode permet de calculer exactement la partie oscillante dans le milieu discret et d'introduire uniquement l'approximation des milieux continus pour l'enveloppe de l'excitation.

On cherche, pour les modes symétriques, des solutions du système (1) sous la forme

$$
W(\ell, m)=\varepsilon F_{\ell}(t) \cos q m a e^{i \theta}+c . c .+\ldots
$$

où $\theta=(k \ell a-\omega t)$ est la phase, $\varepsilon \ll 1$ (un paramètre d'amplitude), $k$ et $\omega$ sont liés par la relation de

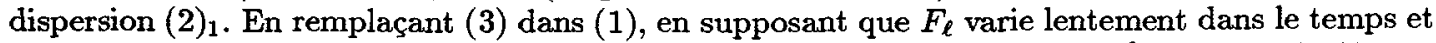
l'espace, et en effectuant le changement de référentiel $s=\varepsilon\left(X-V_{g} t\right), \tau=\varepsilon^{2} t$ où $V_{g}=\partial \omega / \partial k$ est la vitesse de groupe de la porteuse, on obtient à l'ordre d'approximation le plus bas une équation de Schrödinger non linéaire $(N L S)$ de type cubique qui régit l'évolution de $F_{\ell}(t)=F(s, \tau)$

$$
\begin{gathered}
-i F_{\tau}+P F_{s s}+Q|F|^{2} F=0 \\
P=-\frac{1}{2}\left(\partial V_{g} / \partial k\right), Q=(18 B / \omega M)\left[\sin ^{4} \frac{1}{2} k a+\sin ^{4} \frac{1}{2} q a\right] .
\end{gathered}
$$

Les solutions de l'équation $N L S$ dépendent du signe du produit $P Q$. Ici on remarque que le signe de $P Q$ dépend du mode considéré $p$, du nombre d'onde de la porteuse (lorsque $p \geq 2$ ) et du signe du coefficient non linéaire $B$. En particulier lorsque $P Q<0$ l'équation $N L S$ admet des 
solutions enveloppes trous ou solitons obscurs [8] qui se manifestent sous la forme d'une oscillation d'amplitude maximale constante sauf dans une région localisée où l'enveloppe s'atténue en formant un pulse inversé (fig. 5). En revenant aux variables d'origine les solutions de (1) sous forme de solitons obscurs bidimensionnels s'écrivent :

$$
W(\ell, m)=W_{\max } \cos q m a\left[1-\alpha^{2} \operatorname{sech}^{2}\left(\left(\ell a-v_{e} t\right) / L_{e}\right)\right]^{1 / 2} \exp i(K \ell a-\Omega t+\Phi(\ell a, t))
$$

avec

$$
\begin{aligned}
L_{e}= & (1 / \varepsilon \alpha)\left[|2 P / Q| \rho_{0}\right]^{1 / 2}, v_{e}=V_{g}-\varepsilon P c \\
K= & k+\varepsilon \frac{1}{2} c, W_{\max }=\varepsilon \rho_{0}^{1 / 2}=\left(1 / \alpha L_{e}\right)[|2 P / Q|]^{1 / 2}, \\
\Omega= & \omega+\varepsilon \frac{1}{2} c V_{g}-\varepsilon^{2}\left(\frac{1}{4} c^{2} P+|Q / 2 P| \rho_{0}\left(3-\alpha^{2}\right)\right), \\
\Phi(\ell a, t)= & \left(\left[1-\alpha^{2}\right]^{1 / 2} / \alpha\right)\left(\left(\ell a-v_{e} t\right) / L_{e}\right)+ \\
& +\operatorname{Arctg}\left\{\left(\alpha /\left[1-\alpha^{2}\right]^{1 / 2}\right) \operatorname{th}\left(\left(\ell a-v_{e} t\right) / L_{e}\right)\right\}
\end{aligned}
$$

où $\rho_{0}$ et $c$ sont des paramètres arbitraires et $0 \leq \alpha \leq 1$ régle la profondeur de modulation.

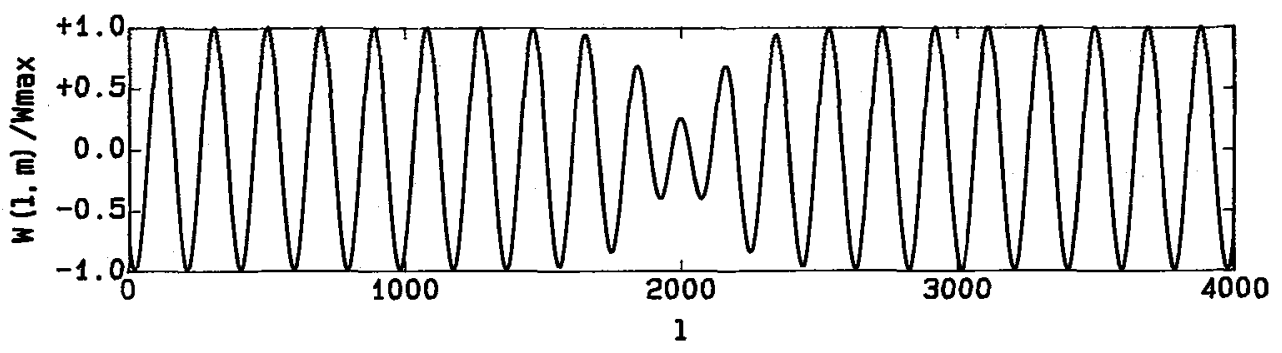

Fig. 5 : Evolution de l'amplitude normalisée, à $m$ fixé, d'un soliton obscur en fonction $d u$ nombre de cellule $\ell$ ( $a=4.08510^{-8} \mathrm{~cm}, M=7.16210^{-22} \mathrm{~g}, A=1.85010^{4} \mathrm{dyn} / \mathrm{cm}$,

$\left.B=-1.40810^{20} \mathrm{dyn} / \mathrm{cm}^{3}, k=0.01 \pi / a, p=0, H=20, L_{e}=200 a, \alpha=0.968, W_{\max }=0.02 a\right)$,

\section{REFERENCES}

[1] M. B. LAKE, H.C. YUEN, H. RUNGALDIER and W.E. FERGUSON, J. Fluid Mech., 83, 49-74 (1977).

[2] L. MOLLENAUER, F. STOLEN and J.P. GORDON, Phys. Rev. Lett., 45, 1095-1098 (1980).

[3] K. MUROYA, N. SAITOH and S. WATANABE, J. Phys. Soc. Japan, 51, 1024-1029 (1982).

[4] M. PLANAT and M. HOUMMADY, Appl. Phys. Lett., 55, 103-105 (1989).

[5] R.D. MINDLIN, Int. J. Solids Structures, 6, 725-738 (1970).

[6] Y.S. KIVSHAR and E.S. SYRKIN, Phys. Lett. A, 153, 125-153 (1991).

[7] N. FLYTZANIS, St. PNEVMATIKOS and M. REMOISSENET, J. Phys. C Solid State Phys., 18, 4603-4629 (1985).

[8] A. HASEGAWA, Optical Solitons in Fibers, Springer, Berlin (1989). 